\title{
Multidimensional Stability of Subsonic Phase Transitions in a Non-Isothermal Van Der Waals Fluid*
}

\author{
Shuyi Zhang \\ School of Science, Shanghai Institute of Technology, Shanghai, China \\ Email: zh_shuyi@yahoo.com.cn
}

Received February 27, 2012; revised May 22, 2012; accepted May 29, 2012

\begin{abstract}
We show the multidimensional stability of subsonic phase transitions in a non-isothermal van der Waals fluid. Based on the existence result of planar waves in our previous work [1], a jump condition is posed on non-isothermal phase boundaries which makes the argument possible. Stability of planar waves both in one dimensional and multidimensional spaces are proved.
\end{abstract}

Keywords: Non-Isothermal Phase Transitions; Euler Equations; Multidimensional Stability

\section{Introduction}

The motion of a 2-dimensional non-isothermal van der Waals fluid is governed by the following Euler equations

$$
\left\{\begin{array}{l}
\partial_{t} \rho+\nabla \cdot(\rho \boldsymbol{u})=0 \\
\partial_{t}(\rho \boldsymbol{u})+\nabla \cdot(\rho \boldsymbol{u} \otimes \boldsymbol{u}+p)=0 \\
\partial_{t}\left(\rho\left(\frac{1}{2}|\boldsymbol{u}|^{2}+e\right)\right)+\nabla \cdot\left(\rho \boldsymbol{u}\left(\frac{1}{2}|\boldsymbol{u}|^{2}+i\right)\right)=0
\end{array}\right.
$$

where $\nabla=\left(\partial_{x}, \partial_{y}\right)^{T}, \rho$ is the density, $\boldsymbol{u}=(u, v)^{T}$ is the velocity with $|\boldsymbol{u}|^{2}=u^{2}+v^{2}, p$ is the pressure satisfying the following state equation

$$
p(\tau, \theta)=\frac{R \theta}{\tau-b}-\frac{a}{\tau^{2}}(\tau>b),
$$

with $\tau \equiv \rho^{-1}$ being the specific volume, $\theta$ being the temperature, $R$ being the perfect gas constant and $a, b$ being positive constants, $e$ is the specific internal energy given by

$$
e(\tau, \theta)=-\frac{a}{\tau}+C_{v} \theta
$$

and $i$ is the specific enthalpy given by

$$
i=e+p \tau \text {. }
$$

*Project 10901107 supported by National Natural Science Foundation of China.

Project 11101076 supported by National Natural Science Foundation of China.

Supported by "The Shanghai Committee of Science and Technology (11ZR1400200)"
Otherwise, according to the second law of thermodynamics, the specific entropy $s$ and the specific free energy $f$ of the fluid is defined by

$$
s=R \ln (\tau-b)+C_{v} \ln \theta+C_{v}
$$

and

$$
f=-\frac{a}{\tau}-R \theta \ln (\tau-b)-C_{v} \theta \ln \theta
$$

respectively. Regarding $(\rho, u, v, s)$ as independent variables and denoting $U=(\rho, u, v, s)^{T}$,

$$
\begin{gathered}
F_{0}(U)=\left(\begin{array}{c}
\rho \\
\rho u \\
\rho v \\
\rho\left(\frac{1}{2} q^{2}+e\right)
\end{array}\right), \quad F_{1}(U)=\left(\begin{array}{c}
\rho u \\
\rho v^{2}+p \\
\rho u v \\
\rho u\left(\frac{1}{2} q^{2}+i\right)
\end{array}\right), \\
F_{2}(U)=\left(\begin{array}{c}
\rho v \\
\rho u v \\
\rho v^{2}+p \\
\rho v\left(\frac{1}{2} q^{2}+i\right.
\end{array}\right)
\end{gathered}
$$

and

$$
\begin{aligned}
A_{1}(U) & =\left(\nabla_{U} F_{0}(U)\right)^{-1} \nabla_{U} F_{1}(U) \\
& =\left(\begin{array}{cccc}
u & \rho & 0 & 0 \\
c^{2} / \rho & u & 0 & \partial_{s} p(\rho, s) / \rho \\
0 & 0 & u & 0 \\
0 & 0 & 0 & u
\end{array}\right),
\end{aligned}
$$




$$
\begin{aligned}
A_{2}(U) & =\left(\nabla_{U} F_{0}(U)\right)^{-1} \nabla_{U} F_{2}(U) \\
& =\left(\begin{array}{cccc}
v & 0 & \rho & 0 \\
0 & v & 0 & 0 \\
c^{2} / \rho & 0 & v & \partial_{s} p(\rho, s) / \rho \\
0 & 0 & 0 & v
\end{array}\right),
\end{aligned}
$$

where $c^{2}=\partial_{\rho} p(\rho, s)$ is the sound speed, we can rewrite (1) as

$$
\partial_{t} F_{0}(U)+\partial_{x} F_{1}(U)+\partial_{y} F_{2}(U)=0
$$

or

$$
\partial_{t} U+A_{1}(U) \partial_{x} U+A_{2}(U) \partial_{y} U=0 .
$$

When $\theta \in(a / 4 b R, 27 a / 8 b R)$, the state Equation (2) is not monotonic with respect to $\tau$, which means that there exist $\tau_{*}(\theta)$ and $\tau^{*}(\theta)$ such that

$$
\begin{cases}\partial_{\tau} p(\tau, \theta)<0 & \tau \in\left(b, \tau_{*}(\theta)\right) \cup\left(\tau^{*}(\theta),+\infty\right) \\ \partial_{\tau} p(\tau, \theta)>0 & \tau \in\left(\tau_{*}(\theta), \tau^{*}(\theta)\right) .\end{cases}
$$

The fluid is in liquid phase in the region $\left(b, \tau_{*}(\theta)\right)$, while it is in vapor phase in the region $\left(\tau^{*}(\theta),+\infty\right)$. The region $\left(\tau_{*}(\theta), \tau^{*}(\theta)\right)$ is a highly unstable region (spinodal region) where no state can be found in experiments [2]. Due to such monotonicity, subsonic phase transitions can be found in a van der Waals fluid, which is different from the well-known classical nonlinear waves such as shock waves, rarefaction waves and contact discontinuities.

A subsonic phase transition is a discontinuous solution to the Euler Equation (1) with a single discontinuity, which changes phases across the discontinuity and satisfies certain subsonic condition on both sides of the discontinuity. To explain the concept with more detail, let us consider the following planar subsonic phase transition

$$
U(t, x, y)= \begin{cases}U_{-}=\left(\rho_{-}, u_{-}, v_{0}, s_{-}\right) & x<\sigma t \\ U_{+}=\left(\rho_{+}, u_{+}, v_{0}, s_{+}\right) & x>\sigma t,\end{cases}
$$

where $\rho_{ \pm}, u_{ \pm}, v_{0}, s_{ \pm}$are constant states of the flow, $\sigma$ is the constant speed of the discontinuity $\{x=\sigma t\}$ and $\rho_{ \pm}$belong to different phases. The solution (10) satisfies the Rankine-Hugoniot condition

$$
\sigma\left[F_{0}(U)\right]-\left[F_{1}(U)\right]=0,
$$

and the subsonic condition

$$
M_{ \pm}=\left|\frac{u_{ \pm}-\sigma}{c_{ \pm}}\right|<1,
$$

where [.] denotes the difference of a function across the discontinuity $\{x=\sigma t\}, M_{ \pm}$and $c_{ \pm}^{2}=d_{\rho} p\left(\rho_{ \pm}, s_{ \pm}\right)$are the Mach number and the sound speed on each side of the discontinuity $\{x=\sigma t\}$ respectively.

Due to the subsonic property (12), the well-known Lax entropy inequality [3] is violated for subsonic phase transitions. Hence, several admissibility criteria were introduced to select the physical admissible subsonic phase transitions, among which the viscosity capillarity criterion proposed by Slemrod [4] is an important one. Ever since, for a long time, attention has been paid to isothermal phase transitions and related problems with numerous works devoted to such topics. For problems in one dimensional spaces, see [2,4-6] and references therein. For problems in multi-dimensional spaces, see [7-10] and references therein.

Compared with isothermal phase transitions, there is much less knowledge on non-isothermal phase transitions and there are fewer papers available. Slemrod [11] and Grinfeld [12] proved the existence of traveling waves in Lagrange coordinates by Conley index theory. Hattori [13] considered certain cases of the Riemann problem by the entropy rate criterion. Recently, the author [1] proved the existence and structural stability of traveling waves by using the center manifold method, in light of which, we can expect to reveal more insights of multidimensional phase transitions.

The purpose of this paper is to study the multidimensional stability of non-isothermal phase transitions. With straightforward computation, we show that the corresponding linearized initial boundary problem for the planar phase transition satisfies the uniform Lopatinski condition $[14,15]$. Without giving much detail, here we briefly state the main result of this paper

Theorem 1.1 There exists $v_{1}>0$ and $K_{1}>0$ depending on the bounds of $U_{+}$and $\sigma$ given in (10) and $\beta$ given in (18), such that for $0<v<v_{1}$ and $0<K<$ $K_{1}$, the $(v, K)$-admissible phase transition (10) is uniformly stable.

The definitions of the parameters $v, K, \beta$ and $(v, K)$ admissible will be given in Section 2, and the uniform stability will be described in detail in Section 4 .

The paper is arranged as follows. Section 2 is a brief recall of the viscosity capillarity criterion for phase transitions and related existence results of traveling waves. In Section 3, we propose the main problem and prove the stability of phase transitions in one dimensional spaces. The multidimensional stability of phase transitions is presented and proved in Section 4.

For the simplicity of notations, we will need the following quantities in the coming arguments.

$$
p^{I}=\frac{R \theta}{\tau-b}, p^{I I}=-\frac{a}{\tau^{2}},
$$




$$
\begin{gathered}
e^{I}=-\frac{a}{\tau}, e^{I I}=C_{v} \theta, \\
s^{I}=R \ln (\tau-b), s^{I I}=C_{v} \ln \theta+C_{v}, \\
f^{I}=-\frac{a}{\tau}-R \theta \ln (\tau-b), f^{I I}=-C_{v} \theta \ln \theta .
\end{gathered}
$$

Considering the planar subsonic phase transition (10), we denote by $j=\rho_{ \pm}\left(u_{ \pm}-\sigma\right)$ the mass transfer flux, and $\pi=p_{ \pm}+j^{2} \tau_{ \pm}$and $\varepsilon=e_{ \pm}+\pi \tau_{ \pm}-j^{2} \tau_{ \pm}^{2} / 2$. Then, the Rankine-Hugoniot condition (11) and the subsonic condition (12) can be rewritten as

$$
[j]=0,[\pi]=0,[\varepsilon]=0
$$

and

$$
\left|\frac{j^{2}}{d_{\tau} p\left(\tau_{ \pm}, s_{ \pm}\right)}\right|<1
$$

respectively.

\section{Viscosity Capillarity Profiles}

Analogue to the traveling wave method for viscous shocks, the viscosity capillarity criterion is applied to find the planar wave (10) which admits the existence of the following traveling wave

$$
U(\xi)=U\left(\frac{x-\sigma t}{\varepsilon}\right)
$$

satisfying $U( \pm \infty)=U_{ \pm}$and the Navier-Stokes equations

$$
\left\{\begin{array}{l}
\partial_{t} \rho+\nabla \cdot(\rho \boldsymbol{u})=0 \\
\partial_{t}(\rho \boldsymbol{u})+\nabla \cdot(\rho \boldsymbol{u} \otimes \boldsymbol{u}+p)=\varepsilon v \Delta \boldsymbol{u}-\varepsilon^{2} \nabla \Delta\left(\rho^{-1}\right) \\
\partial_{t}\left(\rho\left(\frac{1}{2}|\boldsymbol{u}|^{2}+e\right)\right)+\nabla \cdot\left(\rho \boldsymbol{u}\left(\frac{1}{2} q^{2}+i\right)\right) \\
\quad=\varepsilon v \nabla \cdot(\boldsymbol{u} \nabla \cdot \boldsymbol{u})-\varepsilon^{2} \boldsymbol{u} \cdot \nabla \Delta\left(\rho^{-1}\right)+\varepsilon \kappa \Delta \theta,
\end{array}\right.
$$

where $\Delta=\partial_{x}^{2}+\partial_{y}^{2}$ is the Laplace operator, $\varepsilon v$ is the viscosity coefficient, $\varepsilon^{2}$ is the capillarity coefficient and $\varepsilon \kappa$ is the heat conductivity coefficient with $\varepsilon \geq 0$, $v>0, \kappa>0$. Substituting (15) into (16) and noticing the Rankine-Hugoniot conditions (11), we can derive the following heteroclinic problem for the unknown functions $(\tau(\xi), \theta(\xi))$,

$$
\left\{\begin{array}{l}
\tau^{\prime \prime}=v j \tau^{\prime}+\pi-p(\tau, \theta)-j^{2} \tau \\
\theta^{\prime}=\frac{1}{\kappa}\left(-\frac{\left(\tau^{\prime}\right)^{2}}{2}+e(\tau, \theta)+\pi \tau-\frac{j^{2}}{2} \tau^{2}-\mathcal{E}\right) \\
\tau( \pm \infty)=\tau_{ \pm} \theta( \pm \infty)=\theta_{ \pm},
\end{array}\right.
$$

where the prime ' denotes the derivative of a function with respect to $\xi$.

In order to deal with the above problem by the center manifold method, we proposed the following assumption in [1],

$$
\frac{1}{\kappa}=O\left(\frac{1}{C_{v}}\right) \text { as } C_{v} \rightarrow \infty,(A)
$$

which was later simplified as

$$
\frac{1}{\kappa}=\beta K
$$

with $M$ being a positive constant and $K \equiv \frac{1}{C_{v}}$. Employing the Rankine-Hugoniot conditions (13), the hecteroclinic problem (17) becomes

$$
\left\{\begin{aligned}
& \tau^{\prime \prime}=v j \tau^{\prime}+\pi-p(\tau, \theta)-j^{2} \tau \\
& \theta^{\prime}=j K \beta\left(\frac{\left(\tau^{\prime}\right)^{2}}{2}-\frac{a}{\tau}+\pi \tau-\frac{j^{2}}{2} \tau^{2}\right. \\
&\left.-\left(-\frac{a}{\tau_{-}}+\pi \tau_{-}-\frac{j^{2}}{2} \tau_{-}^{2}\right)\right)+j \beta\left(\theta-\theta_{-}\right) \\
&(\tau, \theta)( \pm \infty)=\left(\tau_{ \pm}, \theta_{ \pm}\right) .
\end{aligned}\right.
$$

Therefore, the admissibility of subsonic phase transitions can be defined by

Definition 2.1 The planar phase transition (10) is admissible if and only if the problem (19) has a solution. The solution $(\tau(\xi), \theta(\xi))$ is called the viscosity capillarity profile, or $(v, K)$-profile for simplicity. The pair $\left(\tau_{-}, \theta_{-}\right),\left(\tau_{+}, \theta_{+}\right)$is called $(v, K)$-admissible.

To state the existence result of $(v, K)$-profile, we will need the following quantities. As usual for fixed $\theta \in$ $(a / 4 b R, 8 a / 27 b R)$, the Maxwell equilibrium $\left\{\tau_{m}(\theta), \tau_{M}(\theta)\right\}$ is defined by the equal area rule

$$
\int_{\tau_{m}(\theta)}^{\tau_{M}(\theta)}\left(p\left(\tau_{m}(\theta), \theta\right)-p(\tau, \theta)\right) \mathrm{d} \tau=0 .
$$

Then there exists a unique point $\tau_{1}(\theta) \in\left(\tau_{m}(\theta),+\infty\right)$, which satisfies that the chord connecting the points $\left(\tau_{1}(\theta), p\left(\tau_{1}(\theta), \theta\right)\right)$ and $\left(\tau_{m}(\theta), p\left(\tau_{m}(\theta), \theta\right)\right)$ is tangent to the graph of $p=p(\tau, \theta)$ at the point $\left(\tau_{1}(\theta), p\left(\tau_{1}(\theta), \theta\right)\right)$. Denote

$$
j_{1}^{2}(\theta)=\frac{p\left(\tau_{1}(\theta), \theta\right)-p\left(\tau_{m}(\theta), \theta\right)}{\tau_{m}(\theta)-\tau_{1}(\theta)} .
$$

When $v=K=0$, the $(0,0)$-profile satisfies

$$
\left\{\begin{array}{l}
\tau^{\prime \prime}=\pi-p(\tau, \theta)-j^{2} \tau \\
\theta^{\prime}=j M\left(\theta-\theta_{-}\right) \\
(\tau, \theta)( \pm \infty)=\left(\tau_{ \pm}, \theta_{ \pm}\right),
\end{array}\right.
$$

which implies $\theta_{-}=\theta_{+}$. Setting $\theta(\xi) \equiv \theta_{-}$, there exists 
$\bar{\tau}\left(\xi ; j, \theta_{-}\right)$satisfying the first equation of (20) by the generalized equal area rule as in [8], which means

$$
\int_{\tau_{-}}^{\tau_{+}}\left(\pi-p\left(\tau, \theta_{-}\right)-j^{2} \tau\right) \mathrm{d} \tau=0 .
$$

Moreover, for every $\theta_{-} \in(4 a / b R, 8 a / 27 b R)$ and $\bar{j}$ $\left(0<\bar{j}^{2} \leq j_{1}^{2}\left(\theta_{-}\right)\right)$, a unique pair $\left(\bar{\tau}_{-}\left(\bar{j}, \theta_{-}\right), \bar{\tau}_{+}\left(\bar{j}, \theta_{-}\right)\right)$ can be found such that $\left(\bar{\tau}_{-}, \theta_{-}\right)$and $\left(\bar{\tau}_{+}, \theta_{-}\right)$can be connected by the $(0,0)$-profile with the parameters $j$ and $\theta_{-}$.

Based on the existence of $(0,0)$-profile, the following theorem shows the existence of $(v, K)$-profile for small $v$ and small $K$ in [1].

Theorem 2.1 For every $\theta_{-} \in(a / 4 b R, 8 a / 27 b R)$ and $0<\bar{j}^{2} \leq j_{1}^{2}\left(\theta_{-}\right)$, there exist $v_{0}>0, K_{0}>0$ and neighborhoods $\mathcal{J}_{0}, \mathcal{V}_{0}, \mathcal{T}_{0}$ of $\bar{j},\left(\bar{\tau}_{-}\left(\bar{j}, \theta_{-}\right), \bar{\tau}_{+}\left(\bar{j}, \theta_{-}\right)\right), \theta_{-}$ respectively, such that, for $(j, v, K) \in \mathcal{J}_{0} \times\left[0, v_{0}\right] \times\left[0, K_{0}\right]$, there are unique pair $\left(\tau_{-}, \tau_{+}\right) \in \mathcal{V}_{0}$ and $\theta_{+} \in \mathcal{T}_{0}$, for which $\left(\tau_{-}, \theta_{-}\right)$and $\left(\tau_{+}, \theta_{+}\right)$are $(v, K)$-admissible with the parameters $j$ and $\theta_{-}$.

Moreover, an additional jump condition can be derived for (10), which plays an essential role in the study of the stability of phase transitions. In the isothermal case [4], due to the subsonic condition (12), the Rankine-Hugoniot condition (11) is not sufficient to guarantee the wellposedness of the boundary value problem for phase transitions, which is also the situation that we encounter in the study of non-isothermal case.

By multiplying the first equation of (19) with $\tau^{\prime}(\xi)$ and integrating from $-\infty$ to $+\infty$ with respect to $\xi$, we get the following jump condition on the phase boundary

$$
\begin{aligned}
{\left[f^{I}+\pi \tau-\frac{j^{2}}{2} \tau^{2}\right]=} & -v a\left(j, \theta_{-} ; \nu, K\right) \\
& -K b\left(j, \theta_{-} ; v, K\right)
\end{aligned}
$$

where

$$
\begin{aligned}
& a\left(j, \theta_{-} ; v, K\right)=j \int_{-\infty}^{+\infty}\left(\tau^{\prime}(\xi)\right)^{2} \mathrm{~d} \xi, \\
& b\left(j, \theta_{-} ; v, K\right)=\int_{-\infty}^{+\infty} \Theta^{\prime}(\xi) s^{I}(\xi) \mathrm{d} \xi
\end{aligned}
$$

with $\Theta(\xi)=\left(\theta(\xi)-\theta_{-}\right) / K, s^{I}(\xi)=R \ln (\tau(\xi)-b)$ and $(\tau(\xi), \theta(\xi))$ being the $(v, K)$-profile with the parameters $j$ and $\theta_{-}$.

Remark 2.1 $\Theta(\xi)$ is a bounded function which also possesses a uniform limit as $K \rightarrow 0^{+}, v \rightarrow 0^{+}$. Indeed, from the second equation of (19), we have

$$
\left\{\begin{array}{l}
\Theta^{\prime}=\beta j(h(\tau)+\Theta) \\
\Theta(+\infty)=-h\left(\tau_{+}\right), \quad \Theta(-\infty)=0,
\end{array}\right.
$$

where

$$
h(\tau)=\frac{\left(\tau^{\prime}\right)^{2}}{2}-\frac{a}{\tau}+\pi \tau-\frac{j^{2}}{2} \tau^{2}-\left(-\frac{a}{\tau_{-}}+\pi \tau_{-}-\frac{j^{2}}{2} \tau_{-}^{2}\right) .
$$

Simple calculation yields

$$
\Theta(\xi)= \begin{cases}\int_{-\infty}^{\xi} \beta j h(\tau(\zeta)) e^{\beta j(\xi-\zeta)} \mathrm{d} \zeta & j<0 \\ -\int_{\xi}^{+\infty} \beta j h(\tau(\zeta)) e^{\beta j(\xi-\zeta)} \mathrm{d} \zeta-h\left(\tau_{+}\right) & j \geq 0 .\end{cases}
$$

When $K \rightarrow 0^{+}, v \rightarrow 0^{+}, \Theta$ has a uniform limit

$\lim _{\substack{K \rightarrow 0^{+} \\ v \rightarrow 0^{+}}} \Theta(\xi)= \begin{cases}\int_{-\infty}^{\xi} \beta j h(\bar{\tau}(\zeta)) e^{\beta j(\xi-\zeta)} \mathrm{d} \zeta & j<0 \\ -\int_{\xi}^{+\infty} \beta j h(\bar{\tau}(\zeta)) e^{\beta j(\xi-\zeta)} \mathrm{d} \zeta-h\left(\bar{\tau}_{+}\right) & j \geq 0,\end{cases}$

where $\bar{\tau}(\xi)$ is the $(0,0)$-profile.

Moreover, from the following jump conditions

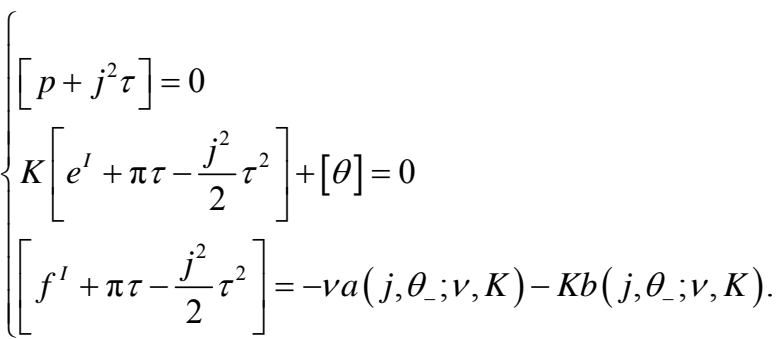

the functions $\left(\tau_{+}, \tau_{-}, \theta_{+}\right)$of $\left(j, \theta_{-}, v, K\right)$ can be determined by the implicity function theorem for $(v, K)$ near $(0,0)$ and every $\left(j, \theta_{-}\right)$satisfying the conditions given in Theorem 2.1. The following identities can be easily verified

$$
\begin{aligned}
\partial_{j}\left(\begin{array}{l}
\bar{\tau}_{+} \\
\bar{\tau}_{-} \\
\bar{\theta}_{+}
\end{array}\right) & =\left(\begin{array}{c}
\frac{j[\bar{\tau}]}{C_{+}^{2}} \\
\frac{j[\bar{\tau}]}{C_{-}^{2}} \\
0 \\
\bar{\theta}_{+}
\end{array}\right), \partial_{\theta_{-}}\left(\begin{array}{l}
\bar{\tau}_{+} \\
\bar{\tau}_{-} \\
\bar{\theta}_{+}
\end{array}\right)=\left(\begin{array}{c}
\frac{\bar{p}_{+}^{I}}{\overline{\theta_{+} C_{+}^{2}}}-\frac{\bar{s}_{+}^{I}+\bar{s}_{-}^{I}}{C_{+}^{2}[\bar{\tau}]} \\
\frac{\bar{p}_{-}^{I}}{\theta_{-} C_{-}^{2}}-\frac{\bar{s}_{+}^{I}+\bar{s}_{-}^{I}}{C_{-}^{2}[\bar{\tau}]} \\
1
\end{array}\right), \\
\partial_{v}\left(\begin{array}{c}
\bar{\tau}_{+} \\
\bar{\tau}_{-} \\
\bar{\theta}_{+}
\end{array}\right) & =\left(\begin{array}{c}
\frac{a_{0}\left(j, \theta_{-}\right)}{C_{+}^{2}[\bar{\tau}]} \\
\frac{a_{0}\left(j, \theta_{-}\right)}{C_{-}^{2}[\bar{\tau}]} \\
0
\end{array}\right), \partial_{K}\left(\begin{array}{c}
\frac{b_{0}\left(j, \theta_{-}\right)}{C_{+}^{2}[\bar{\tau}]} \\
\bar{\tau}_{-} \\
\bar{\theta}_{+}
\end{array}\right)=\left(\begin{array}{c}
b_{0}\left(j, \theta_{-}\right) \\
C_{-}^{2}[\bar{\tau}] \\
0
\end{array}\right),
\end{aligned}
$$

where - denotes the value of a function for $(v, K)=$ $(0,0)$ and

$$
\begin{aligned}
& a_{0}\left(j, \theta_{-}\right)=a\left(j, \theta_{-} ; 0,0\right), \\
& b_{0}\left(j, \theta_{-}\right)=b\left(j, \theta_{-} ; 0,0\right),
\end{aligned}
$$




$$
C_{ \pm}^{2}=-\left(\partial_{\tau} p\left(\bar{\tau}_{ \pm}, \bar{\theta}_{ \pm}\right)+j^{2}\right) .
$$

\section{Linearized Problems and One Dimensional Stability}

In this section, we propose the nonlinear problem for a multidimensional subsonic phase transition and derive the corresponding linearized problem. Then we prove the 1-dimensional stability for the linear problem.

\subsection{Linearized Problems}

Endow the Euler Equation (1) with the following initial data

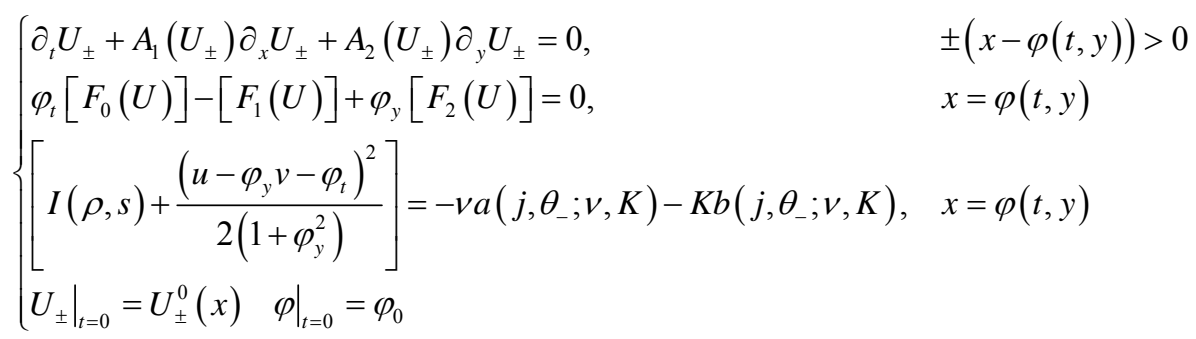

where the third equation is a reformulation of the jump condition $(21)$ with $I(\rho, s)=f^{I}+p \tau$,

$$
\begin{gathered}
j=\rho_{ \pm}\left(u_{ \pm}-\varphi_{y} v_{ \pm}-\varphi_{t}\right) /\left.\sqrt{1+\varphi_{y}^{2}}\right|_{x=\varphi(y, t)}, \\
a\left(j, \theta_{-} ; v, K\right)=j \int_{-\infty}^{+\infty}\left(\tau^{\prime}(\xi)\right)^{2} \mathrm{~d} \xi, \quad b\left(j, \theta_{-} ; v, K\right)=\int_{-\infty}^{+\infty} \Theta^{\prime}(\xi) s^{I}(\xi) \mathrm{d} \xi,
\end{gathered}
$$

and $\tau(\xi ; j, v, K), \theta(\xi ; j, v, K)$ satisfying

$$
\left\{\begin{array}{l}
\tau^{\prime \prime}=v j \tau^{\prime}+\pi-p(\tau, \theta)-j^{2} \tau \\
\theta^{\prime}=j K \beta\left(\frac{\left(\tau^{\prime}\right)^{2}}{2}-\frac{a}{\tau}+\pi \tau-\frac{j^{2}}{2} \tau^{2}-\left(-\frac{a}{\tau_{-}}+\pi \tau_{-}-\frac{j^{2}}{2} \tau_{-}^{2}\right)\right)+j \beta\left(\theta-\theta_{-}\right) . \\
(\tau, \theta)( \pm \infty)=\left.\left(\tau_{ \pm}, \theta_{ \pm}\right)\right|_{x=\varphi(t, y)}
\end{array}\right.
$$

Following [15], we introduce the following transformation to map the free boundary $\{x=\varphi(t, y)\}$ into a fixed boundary $\{\tilde{x}=0\}$

Then the problem (26) becomes

$$
\left\{\begin{array}{l}
\tilde{x}= \pm(x-\varphi(t, y)), \quad \pm(x-\varphi(t, y))>0 \\
\tilde{y}=y \\
\tilde{t}=t \\
\tilde{U}(\tilde{t}, \tilde{x}, \tilde{y})=U(t, x, y)
\end{array}\right.
$$

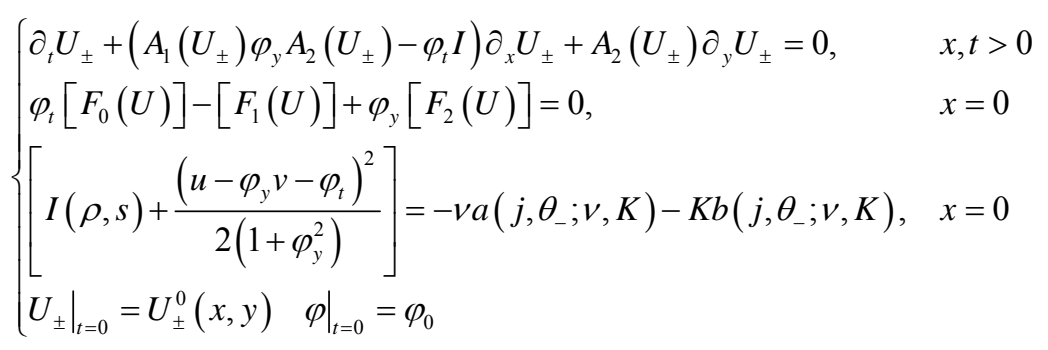


where we have dropped the tildes for simplicity of notations.

Consider the perturbation, $\left(U_{+}^{\varepsilon}, U_{-}^{\varepsilon}, \varphi^{\varepsilon}\right)$, of the planar phase transition (10), which satisfies the problem (26) and $\left.\left(U_{+}^{\varepsilon}, U_{-}^{\varepsilon}, \varphi^{\varepsilon}\right)\right|_{\varepsilon=0}=\left(U_{+}, U_{-}, \sigma x\right)$. Denote

$\left(V_{+}, V_{-}, \psi\right)=\left.\frac{\mathrm{d}}{\mathrm{d} \varepsilon}\left(U_{+}^{\varepsilon}, U_{-}^{\varepsilon}, \varphi^{\varepsilon}\right)\right|_{\varepsilon=0}$. Then, the following linearized problem for the unknowns $\left(V_{+}, V_{-}, \psi\right)$ can be derived from (26),

$$
\begin{cases}\partial_{t} V_{ \pm} \pm\left(A_{1}\left(U_{ \pm}\right)-\sigma I\right) \partial_{x} V_{ \pm}+A_{2}\left(U_{ \pm}\right) \partial_{y} V_{ \pm}=f_{ \pm}, & x, t>0 \\ b_{0} \psi_{t}+b_{1} \psi_{y}+\mathcal{M}_{+} V_{+}+\mathcal{M} V_{-}=g & x=0 \\ \left.\left(V_{+}, V_{-}, \psi\right)\right|_{t<0} \quad \text { vanish } & \end{cases}
$$

where

$$
\begin{gathered}
b_{0}=\left(\begin{array}{c}
{\left[F_{0}(U)\right]} \\
{[u]+\tilde{\gamma} \rho_{-}}
\end{array}\right), b_{1}=\left(\begin{array}{c}
{\left[F_{2}(U)\right]} \\
\left([u]+\tilde{\gamma} \rho_{-}\right) v_{0}
\end{array}\right), \\
\mathcal{M}_{+}=\left(\begin{array}{c}
\sigma F_{0^{\prime}}\left(U_{+}\right)-F_{1^{\prime}}\left(U_{+}\right) \\
l_{+}
\end{array}\right), \\
\mathcal{M}_{-}=\left(\begin{array}{c}
F_{1^{\prime}}\left(U_{-}\right)-\sigma F_{0^{\prime}}\left(U_{-}\right) \\
l_{-}
\end{array}\right)
\end{gathered}
$$

with

$$
\begin{aligned}
l_{+}= & \left(-\frac{\partial I_{+}}{\partial \rho_{+}}, \sigma-u_{+}, 0,-\frac{\partial I_{+}}{\partial s_{+}}\right), \\
l_{-}= & \left(\frac{\partial I_{-}}{\partial \rho_{-}}-\tilde{\gamma}\left(u_{-}-\sigma\right)-\hat{\gamma} \frac{\partial \theta_{-}}{\partial \rho_{-}},\right. \\
& \left.u_{-}-\sigma-\tilde{\gamma} \rho_{-}, 0, \frac{\partial I_{-}}{\partial s_{-}}-\hat{\gamma} \frac{\partial \theta_{-}}{\partial s_{-}}\right)
\end{aligned}
$$

and

$$
\tilde{\gamma}=v \partial_{j} a+K \partial_{j} b, \quad \hat{\gamma}=v \partial_{\theta_{-}} a+K \partial_{\theta_{-}} b .
$$

Remark 3.1 Simple calculation yields

$$
\begin{gathered}
\frac{\partial I}{\partial \rho}=c^{2} \tau-K \tau^{2} p^{I} s^{I}, \frac{\partial I}{\partial s}=K\left(\tau p^{I}-\theta s^{I}\right), \\
\frac{\partial \theta}{\partial \rho}=K \tau^{2} p^{I}, \frac{\partial \theta}{\partial s}=K \theta, \text { and } \partial_{s} p(\rho, s)=K p^{I} .
\end{gathered}
$$

Noticing that the boundary conditions of (29) involve the quantities $\partial_{j} a, \partial_{j} b, \partial_{\theta_{-}} a$ and $\partial_{\theta_{-}} b$, we will need the following lemma to deal with these quantities.

Lemma 3.1 For all $(v, K) \in\left[0, v_{0}\right] \times\left[0, K_{0}\right]$, the functions $a\left(j, \theta_{-} ; v, K\right)$ and $b\left(j, \theta_{-} ; v, K\right)$ are continuously differentiable. Moreover, their derivatives are continuous with respect to $(v, K)$ at $(0,0)$ and are bounded de- pending on the bounds of $U_{ \pm}$and $\sigma$ given in (10) and the constant $M$ given in (18). There exists $\alpha>0$ such that for all $j \in \mathcal{J}$

$$
\lim _{(v, K) \rightarrow(0,0)} \frac{\partial}{\partial j} a\left(j, \theta_{-} ; v, K\right) \geq \alpha>0 .
$$

Proof. The estimate (30) is immediate from Lemma 2 in [7], once we prove the continuity of $\partial_{j} a\left(j, \theta_{-} ; v, K\right)$. Let us show the continuity of $\partial_{j} a$ and $\partial_{j} b$. Differentiating (21) with respect to $j$, we get

$$
-s_{+}^{I} \frac{\partial \theta_{+}}{\partial j}+\frac{\partial \pi}{\partial j}[\tau]-j^{2}\left[\tau^{2}\right]=-v \partial_{j} a-K \partial_{j} b .
$$

By Taylor's formula, we have

$$
\begin{gathered}
s_{+}^{I}=\bar{s}_{+}^{I}+o(1), \\
\frac{\partial \theta_{+}}{\partial j}=\frac{\partial^{2} \bar{\theta}_{+}}{\partial j \partial K} K+o(1) r, \\
\frac{\partial \pi}{\partial j}=\frac{\partial \bar{\pi}}{\partial j}+\frac{\partial^{2} \bar{\pi}}{\partial j \partial v} v+\frac{\partial^{2} \bar{\pi}}{\partial j \partial K} K+o(1) r,
\end{gathered}
$$

where $r=\sqrt{v^{2}+K^{2}}$ and $o(1)$ is an infinitesimal as $r$ goes to zero. Substituting the above identities into (31) and employing the calculations (23), we get

$$
-v \partial_{j} a_{0}-K \partial_{j} b_{0}+o(r)=-v \partial_{j} a-K \partial_{j} b,
$$

which implies

$$
\lim _{(v, K) \rightarrow(0,0)} \partial_{j} a=\partial_{j} a_{0}, \lim _{(v, K) \rightarrow(0,0)} \partial_{j} b=\partial_{j} \cdot b_{0} .
$$

Similar arguments yield the continuity of $\partial_{\theta} a$ and $\partial_{\theta_{-}} b$ as the following

$$
\lim _{(v, K) \rightarrow(0,0)} \partial_{\theta_{-}} a=\partial_{\theta_{-}} a_{0}, \lim _{(v, K) \rightarrow(0,0)} \partial_{\theta_{-}} b=\partial_{\theta_{-}} b_{0} .
$$

\subsection{One Dimensional Stability}

The one dimensional stability concerns the stability of the problem (29) without terms of $y$-derivatives, namely,

$$
\begin{cases}\partial_{t} V_{ \pm} \pm\left(A_{1}\left(U_{ \pm}\right)-\sigma I\right) \partial_{x} V_{ \pm}=f_{ \pm}, & x, t>0 \\ b_{0} \psi_{t}+\mathcal{M}_{+} V_{+}+\mathcal{M} V_{-}=g & x=0 \\ \left.\left(V_{+}, V_{-}, \psi\right)\right|_{t<0} \text { vanish. } & \end{cases}
$$

The following theorem shows the stability of planar phase transitions in one dimensional spaces.

Theorem 3.2 There exists $K_{0}>0$ depending on the bounds of $U_{ \pm}$and $\sigma$ given in (10) and the constant $\beta$ given in (18), such that for any fixed $0<v \leq v_{0} \quad\left(v_{0}\right.$ is given in Lemma 3.1.) and $0<K \leq K_{0}$, the subsonic phase transition (10) is stable with respect to perturbations in the $x$-direction, which means the problem (32) is well-posed.

Proof. The main idea of the proof is to show that the 
boundary values of outgoing characteristics and the free boundary can be determined by the boundary conditions, for which we need to investigate the eigenvalues and the eigenvectors of the matrix $A_{1}\left(U_{ \pm}\right)-\sigma I$. The eigenvalues of $A_{1}\left(U_{ \pm}\right)-\sigma I$ are

$$
\lambda_{1}^{ \pm}=u_{ \pm}-c_{ \pm}-\sigma, \lambda_{3}^{ \pm}=u_{ \pm}+c_{ \pm}-\sigma .
$$

of multiplicity 1 and

$$
\lambda_{2}^{ \pm}=u_{ \pm}-\sigma
$$

of multiplicity 2 . The corresponding right eigenvectors are

$$
r_{1}^{ \pm}=\left(1,-c_{ \pm} \tau_{ \pm}, 0,0\right)^{T}, r_{3}^{ \pm}=\left(1, c_{ \pm} \tau_{ \pm}, 0,0\right)^{T}
$$

and

$$
r_{21}^{ \pm}=(0,0,1,0)^{T}, r_{22}^{ \pm}=\left(-K p_{ \pm}^{I}, 0,0, c_{ \pm}^{2}\right)^{T}
$$

respectively.

Denote by

$$
V_{ \pm}=v_{1}^{ \pm} r_{1}^{ \pm}+v_{21}^{ \pm} r_{21}^{ \pm}+v_{22}^{ \pm} r_{22}^{ \pm}+v_{3}^{ \pm} r_{3}^{ \pm}
$$

the decompositions of $V_{ \pm}$on the bases $\left\{r_{1}^{ \pm}, r_{21}^{ \pm}, r_{22}^{ \pm}, r_{3}^{ \pm}\right\}$ respectively. Since the mass transfer flux $j=\rho_{ \pm}\left(u_{ \pm}-\sigma\right)$ is nonzero, we assume $j>0$. Then the subsonic condition (14) becomes

$$
\lambda_{1}^{ \pm}<0<\lambda_{2}^{ \pm}<\lambda_{3}^{ \pm} .
$$

Accordingly, we rewrite the boundary condition of (32) as

$$
\begin{gathered}
\left(b_{0}, \mathcal{M}_{+} r_{21}^{+}, \mathcal{M}_{+} r_{22}^{+}, \mathcal{M}_{+} r_{3}^{+}, \mathcal{M}_{-} r_{1}^{-}\right)\left(\begin{array}{c}
\psi_{t} \\
v_{21}^{+} \\
v_{22}^{+} \\
v_{3}^{+} \\
v_{1}^{-}
\end{array}\right) \\
=g-\left(\mathcal{M}_{+} r_{1}^{+}, \mathcal{M}_{-} r_{21}^{-}, \mathcal{M}_{-} r_{22}^{-}, \mathcal{M}_{-} r_{3}^{-}\right)\left(\begin{array}{c}
v_{1}^{+} \\
v_{21}^{-} \\
v_{22}^{-} \\
v_{3}^{-}
\end{array}\right)
\end{gathered}
$$

to separate the outgoing characteristics together with the free boundary from the incoming characteristics. The necessary and sufficient condition for the well-posedness of the problem (32) is that the determinant

$$
\mathcal{D} \equiv \operatorname{det}\left(b_{0}, \mathcal{M}_{+} r_{21}^{+}, \mathcal{M}_{+} r_{22}^{+}, \mathcal{M}_{+} r_{3}^{+}, \mathcal{M}_{-} r_{1}^{-}\right)
$$

does not vanish. Direct computation yields

$$
\begin{aligned}
\mathcal{D}=j^{2} \theta_{+} c_{+}^{2} \lambda_{3}^{+} \lambda_{1}^{-}\left|\begin{array}{ccc}
-\frac{[\tau]}{\tau_{+} \tau_{-}} & -1 & 1 \\
0 & -\lambda_{3}^{+} & \lambda_{1}^{-} \\
j[\tau]+\frac{\nu \partial_{j} a}{\tau_{-}} & -\tau_{+} c_{+} & -\left(\tau_{-} c_{-}+v \partial_{j} a\right)
\end{array}\right| \\
+O(1) K
\end{aligned}
$$

where $O(1)$ denotes a bounded term depending on the bounds of $U_{ \pm}, \sigma$ given in (10) and $M$ given in (19). The determinant on the right side of (35) takes the value

$$
\frac{[\tau]^{2}}{\tau_{-} \tau_{+}} \lambda_{3}^{+} \lambda_{1}^{-}-v \partial_{j} a\left(\frac{c_{-}}{\tau_{-}}+\frac{c_{+}}{\tau_{+}}\right)<0
$$

for $0<v \leq v_{0}$ with $v_{0}$ given in Lemma 3.1.

Therefore, we can find $K_{0}$ depending on the bounds of $U_{ \pm}, \sigma$ given in (10) and the constant $\beta$ given in (18) such that for $0<K \leq K_{0}$ and $0<v \leq v_{0}, \Delta$ the problem (32) is well-posed. Similar arguments can be carried out for the case $j<0$.

\section{Multidimensional Stability}

First let us introduce the uniform stability in [15] and state the main result in detail. Denote by

$$
V=\left(V_{+}, V_{-}\right)^{T}
$$

and

$$
\hat{V}(s, \omega, x)=\frac{1}{(2 \pi)^{2}} \int_{0}^{\infty} \int_{-\infty}^{\infty} e^{-(s t+i \omega y)} V(t, x, y) \mathrm{d} y \mathrm{~d} t
$$

the Laplace-Fourier transform of $V$ in $(t, y)$-variables with $\operatorname{Re} s>0$. Then, from (29) we know that $\hat{V}$ satisfies

$$
\frac{\partial \hat{V}}{\partial x}=B(s, \omega) \hat{V}+\hat{f}
$$

where

$$
B(s, \omega)=\left(\begin{array}{cc}
-\left(A_{1}\left(U_{+}\right)-\sigma I\right)^{-1}\left(s I+i \omega A_{2}\left(U_{+}\right)\right) & 0 \\
0 & \left(A_{1}\left(U_{-}\right)-\sigma I\right)^{-1}\left(s I+i \omega A_{2}\left(U_{-}\right)\right)
\end{array}\right)
$$


and

$$
\hat{f}=\left(\left(A_{1}\left(U_{+}\right)-\sigma I\right)^{-1} \hat{f}_{+},-\left(A_{1}\left(U_{-}\right)-\sigma I\right)^{-1} \hat{f}_{-}\right)^{T} .
$$

Denote by $\left\{\lambda_{j}\right\}_{j=1}^{l}$ all the distinct eigenvalues of $B(s, \omega)$ with multiplicity being $m_{j}$. Obviously, we have

$$
\mathbb{C}^{8}=\bigoplus_{j=1}^{l} \operatorname{Ker}\left[\left(\lambda_{j} I-B(s, \omega)\right)^{m_{j}}\right] .
$$

$$
\begin{aligned}
& \text { Introduce } \\
& E^{+}(s, \omega) \\
& =\left\{v_{j} \in \operatorname{Ker}\left[\left(\lambda_{j} I-B(s, \omega)\right)^{m_{j}}\right] \mid \operatorname{Re} \lambda_{j}<0,1 \leq j \leq l\right\}
\end{aligned}
$$

the space of boundary values of all bounded solutions of the special form

$$
\hat{V}(s, \omega, x)=\sum_{\operatorname{Re} \lambda_{j}<0} e^{\lambda_{j} x} \sum_{p=0}^{m_{j}-1} \frac{x^{p}}{p !}\left(\lambda_{j} I-B(s, \omega)\right)^{p} v_{j}
$$

to (36) with $\hat{f} \equiv 0$.

Thus, we can state the uniform stability result in detail

$$
\frac{\partial \hat{V}_{ \pm}}{\partial x}= \pm \frac{1}{d_{ \pm}^{2}}\left(\begin{array}{cccc}
\tilde{s}\left(u_{ \pm}-\sigma\right) & -\tilde{s} \rho_{ \pm} & i \omega \rho_{ \pm}\left(u_{ \pm}-\sigma\right) & K \tilde{s} p_{ \pm}^{I} \\
-\frac{\tilde{s} c_{ \pm}^{2}}{\rho_{ \pm}} & \tilde{s}\left(u_{ \pm}-\sigma\right) & -i \omega c_{ \pm}^{2} & -\frac{K \tilde{s} p_{ \pm}^{I}}{\rho_{ \pm}} \\
-\frac{i \omega c_{ \pm}^{2} d_{ \pm}^{2}}{\rho_{ \pm}\left(u_{ \pm}-\sigma\right)} & 0 & -\frac{\tilde{s} d_{ \pm}^{2}}{u_{ \pm}-\sigma} & -\frac{i \omega d_{ \pm}^{2} K p_{ \pm}^{I}}{\rho_{ \pm}\left(u_{ \pm}-\sigma\right)} \\
0 & 0 & 0 & -\frac{\tilde{s} d_{ \pm}^{2}}{u_{ \pm}-\sigma}
\end{array}\right) \hat{V}_{ \pm}
$$

where $\tilde{s}=s+i \omega v_{0}$ and $d_{ \pm}=\sqrt{c_{ \pm}^{2}-\left(u_{ \pm}-\sigma\right)^{2}}$.

As in [15], if we introduce the transformation

$$
\hat{Z}_{ \pm}=\left(\begin{array}{cccc}
0 & 0 & 1 & 0 \\
\frac{c_{ \pm}}{\sqrt{2} \rho_{ \pm}} & \frac{1}{\sqrt{2}} & 0 & \frac{K p_{ \pm}^{I}}{\sqrt{2} \rho_{ \pm} c_{ \pm}} \\
\frac{c_{ \pm}}{\sqrt{2} \rho_{ \pm}} & -\frac{1}{\sqrt{2}} & 0 & \frac{K p_{ \pm}^{I}}{\sqrt{2} \rho_{ \pm} c_{ \pm}} \\
0 & 0 & 0 & 1
\end{array}\right) \hat{V}_{ \pm},
$$

then (38) is equivalent to

$$
\frac{\partial \hat{Z}_{ \pm}}{\partial x}= \pm N_{ \pm}(s, \omega) \hat{Z}_{ \pm}
$$

where

$$
N_{ \pm}(s, \omega)=\left(\begin{array}{cccc}
-\frac{\tilde{s}}{u_{ \pm}-\sigma} & \frac{i \omega c_{ \pm}}{\sqrt{2}\left(u_{ \pm}-\sigma\right)} & -\frac{i \omega c_{ \pm}}{\sqrt{2}\left(u_{ \pm}-\sigma\right)} & 0 \\
-\frac{i \omega c_{ \pm}}{\sqrt{2}\left(u_{ \pm}-\sigma+c_{ \pm}\right)} & -\frac{\tilde{s}}{u_{ \pm}-\sigma+c_{ \pm}} & 0 & 0 \\
-\frac{i \omega c_{ \pm}}{\sqrt{2}\left(u_{ \pm}-\sigma-c_{ \pm}\right)} & 0 & -\frac{\tilde{s}}{u_{ \pm}-\sigma-c_{ \pm}} & 0 \\
0 & 0 & 0 & -\frac{\tilde{s}}{u_{ \pm}-\sigma}
\end{array}\right) .
$$


The eigenvalues of $N_{+}(s, \omega)$ with negative real part for $\operatorname{Re} s>0$ are

$$
\lambda_{1}^{+}=-\frac{\tilde{s}}{u_{+}-\sigma}
$$

of multiplicity 2 and

$$
\lambda_{2}^{+}=\frac{\tilde{s}\left(u_{+}-\sigma\right)-c_{+} \sqrt[+]{\tilde{s}^{2}+\omega^{2} d_{+}^{2}}}{d_{+}^{2}}
$$

of multiplicity 1 , where the $\sqrt[+]{\cdot}$ denotes the positive real part square root of a complex value. The corresponding eigenvectors are

$$
\begin{gathered}
e_{11}^{+}=(0,0,0,1)^{T}, \\
e_{12}^{+}=\left(\sqrt{2} \tilde{s}, i \omega\left(u_{+}-\sigma\right),-i \omega\left(u_{+}-\sigma\right), 0\right)^{T}
\end{gathered}
$$

and

$$
\begin{aligned}
e_{2}^{+}= & \left(-i \sqrt{2} \omega c_{+}, \tilde{s}+\lambda_{2}^{+}\left(u_{+}-\sigma-c_{+}\right),\right. \\
& \left.\tilde{s}+\lambda_{2}^{+}\left(u_{+}-\sigma+c_{+}\right), 0\right)^{T}
\end{aligned}
$$

respectively. The eigenvalue of $-N_{-}(s, \omega)$ with a negative real part for $\operatorname{Re} s>0$ is

$$
\lambda_{3}^{-}=\frac{\tilde{s}\left(\sigma-u_{-}\right)-c_{-} \sqrt[+]{\tilde{s}^{2}+\omega^{2} d_{-}^{2}}}{d_{-}^{2}}
$$

and the corresponding eigenvector is

$$
\begin{aligned}
e_{3}^{-}= & \left(-i \sqrt{2} \omega c_{-}, \tilde{s}-\lambda_{3}^{-}\left(u_{-}-\sigma-c_{-}\right),\right. \\
& \left.\tilde{s}-\lambda_{3}^{-}\left(u_{-}-\sigma+c_{-}\right), 0\right)^{T} .
\end{aligned}
$$

Remark 4.1 The above eigenvalues and eigenvectors can be continuously extended to the case $\operatorname{Re} s \geq 0$. With a little abuse of the notation $\sqrt[+]{\cdot}$, we still use it to denote those extensions of square roots appearing in the case $\operatorname{Re} s=0$.

As in [15], for these vectors, we have

Proposition $4.2\left(e_{11}^{+}, e_{12}^{+}, e_{2}^{+}, e_{3}^{-}\right)$are linearly independent for $|s|^{2}+\omega^{2}=1$ and $\operatorname{Re} s \geq 0$ except at

$$
\left\{(\tilde{s}, \omega) \mid \tilde{s}^{2}=\omega^{2}\left(u_{+}-\sigma\right)^{2} \operatorname{Re} \tilde{s}>0\right\} .
$$

In the above cases, the following proposition help us to find the bases of $E^{+}(s, \omega)$.

Proposition 4.3 1) If $\tilde{s}=\omega\left(u_{+}-\sigma\right)$ and $\operatorname{Re} \tilde{s}>0$, then $\lambda_{1}^{+}=\lambda_{2}^{+}=-\omega$ and the vectors (41), (44) together with the following eigenvectors

$$
\begin{gathered}
e_{12}^{+}=(\sqrt{2},-i, i, 0)^{T} \\
e_{2}^{+}=\left(u_{+}-\sigma+c_{+}, 0, i \sqrt{2}\left(u_{+}-\sigma\right), 0\right)^{T}
\end{gathered}
$$

are linearly independent.
2) If $\tilde{s}=-\omega\left(u_{+}-\sigma\right)$ and $\operatorname{Re} \tilde{s}>0$, then $\lambda_{1}^{+}=\lambda_{2}^{+}=\omega$ and the vectors (41), (44) together with the following eigenvectors

$$
\begin{gathered}
e_{12}^{+}=(\sqrt{2},-i, i, 0)^{T} \\
e_{2}^{+}=\left(u_{+}-\sigma+c_{+}, 0, i \sqrt{2}\left(u_{+}-\sigma\right), 0\right)^{T}
\end{gathered}
$$

are linearly independent.

As in [15], in the critical case $\tilde{s}=0$, the bases of $E^{+}(s, \omega)$ is given by

Proposition 4.4 If $\tilde{s}=0$ and $\omega>0$, then

$$
\lambda_{1}^{+}=0, \lambda_{2}^{+}=-\frac{\omega c_{+}}{d_{+}}, \lambda_{3}^{-}=-\frac{\omega C_{-}}{d_{-}}
$$

and the corresponding eigenvectors

$$
\begin{gathered}
e_{11}^{+}=(0,0,0,1)^{T}, \\
e_{12}^{+}=(0,1,-1,0)^{T}, \\
e_{2}^{+}=\left(1, \frac{i\left(c_{+}-u_{+}+\sigma\right)}{\sqrt{2} d_{+}},-\frac{i\left(c_{+}+u_{+}-\sigma\right)}{\sqrt{2} d_{+}}, 0\right)^{T}, \\
e_{3}^{-}=\left(1,-\frac{i\left(c_{-}-u_{-}+\sigma\right)}{\sqrt{2} d_{-}}, \frac{i\left(c_{-}+u_{-}-\sigma\right)}{\sqrt{2} d_{-}}, 0\right)^{T}
\end{gathered}
$$

are linearly independent.

Combining the above propositions, if we naturally expand the eigenvectors as

$$
\left(\begin{array}{c}
e_{11}^{+} \\
0
\end{array}\right),\left(\begin{array}{c}
e_{12}^{+} \\
0
\end{array}\right),\left(\begin{array}{c}
e_{2}^{+} \\
0
\end{array}\right),\left(\begin{array}{c}
0 \\
e_{3}^{-}
\end{array}\right),
$$

then the bases of $E^{+}(s, \omega)$ are given for $|s|^{2}+\omega^{2}=1$ and $\operatorname{Re} s \geq 0$.

\subsection{Lopatinski Determinant}

Now we can show the uniform stability of the phase transition.

Proof of Theorem 4.1. Taking the Laplace-Fourier transformation on the boundary condition in (29) with $g=0$, multiplying it with the invertible matrix

$$
\left(\begin{array}{ccccc}
1 & 0 & 0 & 0 & 0 \\
-\sigma & 1 & 0 & 0 & 0 \\
-v_{0} & 0 & 1 & 0 & 0 \\
-\frac{q_{+}^{2}}{2}-i_{+} & 0 & -v_{0} & 1 & 0 \\
0 & 0 & 0 & 0 & 1
\end{array}\right),
$$

with $q_{+}^{2}=u_{+}^{2}+v_{0}^{2}$ and introducing the transformation 
(39), we get

$$
c \hat{\psi}+\tilde{\mathcal{M}}_{+} \hat{Z}_{+}+\tilde{\mathcal{M}}_{-} \hat{Z}_{-}=0 \text { on } x=0,
$$

where

$$
c=\left(\begin{array}{c}
{[\rho] \tilde{s}} \\
0 \\
i \omega[p] \\
\left(\sigma \rho_{-}+j\right) j[\tau] \tilde{s} \\
\left([u]+\tilde{\gamma} \rho_{-}\right) \tilde{s}
\end{array}\right), \tilde{\mathcal{M}}_{ \pm}=\left(\begin{array}{c}
\tilde{\mathcal{M}}_{ \pm}^{0} \\
\tilde{I}_{ \pm}
\end{array}\right)
$$

$$
\tilde{\mathcal{M}}_{-}^{0}=\left(\begin{array}{cccc}
0 & \frac{\lambda_{3}^{-}}{\sqrt{2} \tau_{-} c_{-}} & \frac{\lambda_{1}^{-}}{\sqrt{2} \tau_{-} c_{-}} & -\frac{K j \tau_{-} p_{-}^{I}}{c_{-}^{2}} \\
0 & \frac{\lambda_{3}^{-2}}{\sqrt{2} \tau_{-} c_{-}} & \frac{\lambda_{1}^{-2}}{\sqrt{2} \tau_{-} c_{-}} & -\frac{K j \tau_{-}^{2} p_{-}^{I}}{c_{-}^{2}} \\
j & 0 & 0 & 0 \\
0 & \frac{u_{-} \lambda_{3}^{-}}{\sqrt{2} \tau_{-}}+\frac{j \sigma[\tau] \lambda_{3}^{-}}{\sqrt{2} \tau_{-} c_{-}} & -\frac{u_{-} \lambda_{1}^{-}}{\sqrt{2} \tau_{-}}+\frac{j \sigma[\tau] \lambda_{-}^{-}}{\sqrt{2} \tau_{-} c_{-}} & j \theta_{-}-\frac{K j \tau_{-}^{2} p_{-}^{I}[\tau]}{c_{-}^{2}}
\end{array}\right)
$$

and

$$
\begin{gathered}
\tilde{l}_{+}=\left(0,-\frac{\lambda_{3}^{+}}{\sqrt{2}}-\frac{K \tau_{+} p_{+}^{I} s_{+}^{I}}{\sqrt{2} c_{+}},-\frac{\lambda_{1}^{+}}{\sqrt{2}}-\frac{K \tau_{+} p_{+}^{I} s_{+}^{I}}{\sqrt{2} c_{+}}, K\left(\theta_{+} s_{+}^{I}-\frac{K \tau_{+}^{2} p_{+}^{I 2} s_{+}^{I}}{c_{+}^{2}}\right)\right), \\
\tilde{l}_{-}=\left(0, \frac{\lambda_{3}^{-}}{\sqrt{2}}+\frac{K \tau_{-} p_{-}^{I} s_{-}^{I}}{\sqrt{2} c_{-}}-\frac{\tilde{\gamma} \lambda_{3}^{-}}{\sqrt{2} \tau_{-} c_{-}}, \frac{\lambda_{1}^{-}}{\sqrt{2}}-\frac{K \tau_{-} p_{-}^{I} s_{-}^{I}}{\sqrt{2} c_{-}}-\frac{\tilde{\gamma} \lambda_{-}^{-}}{\sqrt{2} \tau_{-} c_{-}}, K\left(\frac{K \tau_{-}^{2} p_{-}^{I 2} s_{-}^{I}}{c_{-}^{2}}+\theta_{-} s_{-}^{I}-+\frac{\tilde{\gamma} \tau_{-} j}{c_{-}^{2}}-\hat{\gamma} \theta_{-}+\frac{K \hat{\gamma} \tau_{-}^{2} p_{-}^{I}}{c_{-}^{2}}\right)\right) .
\end{gathered}
$$

To achieve the result, we need to verify the determinant

$$
\mathcal{L} \equiv \operatorname{det}\left(c, \tilde{\mathcal{M}}_{+} e_{11}^{+}, \tilde{\mathcal{M}}_{+} e_{12}^{+}, \tilde{\mathcal{M}}_{+} e_{2}^{+}, \tilde{\mathcal{M}}_{-} e_{3}^{-}\right)
$$

being nonzero.

Noticing that the eigenvector $e_{11}^{+}$remains the same in all the cases mentioned in Section 4.1, the following simplification can be made to $\mathcal{L}$,

$$
\mathcal{L}=j \theta_{+} \operatorname{det}\left(c, \hat{\mathcal{M}}_{+} e_{12}^{+}, \hat{\mathcal{M}}_{+} e_{2}^{+}, \hat{\mathcal{M}}_{-} e_{3}^{-}\right)+O(1) K,
$$

where $O(1)$ is a bounded term depending on the bounds of $U_{ \pm}, \sigma$ given in (10) and $\beta$ given in (19),

$$
\hat{\mathcal{M}}_{+}=\left(\begin{array}{cccc}
0 & -\frac{\lambda_{3}^{+}}{\sqrt{2} \tau_{+} c_{+}} & -\frac{\lambda_{1}^{+}}{\sqrt{2} \tau_{+} c_{+}} & 0 \\
0 & -\frac{\lambda_{3}^{+2}}{\sqrt{2} \tau_{+} c_{+}} & -\frac{\lambda_{1}^{+2}}{\sqrt{2} \tau_{+} c_{+}} & 0 \\
-j & 0 & 0 & 0 \\
0 & -\frac{\lambda_{3}^{+}}{\sqrt{2}} & -\frac{\lambda_{1}^{+}}{\sqrt{2}} & 0
\end{array}\right) \text {, }
$$

For sufficiently small $K>0$, the determinant $\mathcal{L}$ is nonzero as long as the determinant

$$
\mathcal{L}_{1} \equiv \operatorname{det}\left(c, \hat{\mathcal{M}}_{+} e_{12}^{+}, \hat{\mathcal{M}}_{+} e_{2}^{+}, \hat{\mathcal{M}}_{-} e_{3}^{-}\right)
$$

doesn't vanish. Considering $\mathcal{L}_{1}$, one can find that it is similar to the Lopatinski determinant for the corresponding problem in the isothermal case [7,9]. Noticing Proposition 4.2-4.4, we need to consider the following three cases:

1) $\tilde{s} \neq \omega^{2}\left(u_{+}-\sigma\right)^{2}$ and $\tilde{s} \neq 0$.

We obtain 


$$
\mathcal{L}_{1}=\frac{4 \sqrt{2} j\left(\tilde{s}^{2}-\omega^{2}\left(u_{-}-\sigma\right)^{2}\right)}{\tau_{+} \tau_{-}}\left(I+\left(v \partial_{j} a+K \partial_{j} b\right) I I\right),
$$

where

$$
I=-\frac{j^{2} \tau_{+} \tau_{-}[\tau]}{\tilde{s}} \sqrt[+]{\tilde{s}^{2}+\omega^{2} d_{-}^{2}} \cdot \sqrt[+]{\tilde{s}^{2}+\omega^{2} d_{+}^{2}}-[\tau] c_{+} c_{-} \tilde{s}
$$

and

$$
I I=-\frac{\tau_{-} C_{+}}{[\tau]} \sqrt[+]{\tilde{s}^{2}+\omega^{2} d_{-}^{2}}-\frac{\tau_{+} C_{-}}{[\tau]} \sqrt[+]{\tilde{s}^{2}+\omega^{2} d_{+}^{2}}+\frac{\omega^{2} d_{+}^{2} j \tau_{+}\left(\tilde{s} C_{-}+j \tau_{-}+\sqrt{\tilde{s}^{2}+\omega^{2} d_{-}^{2}}\right)}{\tilde{s}\left(\tilde{s} C_{+}+j \tau_{-}^{+} \sqrt[+]{\tilde{s}^{2}+\omega^{2} d_{+}^{2}}\right)} .
$$

Following [9], we claim that $I+v \partial_{j} a I I$ is nonzero for sufficiently small $v>0$.

In fact, when $\operatorname{Re} \tilde{s}>0$, if $I=0$, then we have

$$
\left(\tilde{s}^{2}+\omega^{2} d_{-}^{2}\right)\left(\tilde{s}^{2}+\omega^{2} d_{+}^{2}\right)=\left(\frac{c_{+} c_{-}}{\left(u_{+}-\sigma\right)\left(u_{-}-\sigma\right)}\right)^{2} \tilde{s}^{4},
$$

which implies

$$
\tilde{s}^{2}=\frac{\omega^{2}\left(d_{+}^{2}+d_{-}^{2}\right) \pm \omega^{2} \sqrt{\left(d_{+}^{2}+d_{-}^{2}\right)^{2}+4 d_{+} d_{-}\left(\left(M_{+} M_{-}\right)^{-2}-1\right)}}{2\left(\left(M_{+} M_{-}\right)^{-2}-1\right)}
$$

with $M_{ \pm}=\frac{u_{ \pm}-\sigma}{c_{ \pm}}$being the Mach numbers. From the subsonic property (12) of the phase transition, we have $0<M_{ \pm}<1$. Due to $\operatorname{Re} \tilde{s}>0$, we deduce that one should take the plus sign in (51), which is not the root of $I$ obviously. Thus, $I$ is always nonzero, which gives that there exist constants $M_{1}>0$, and $v_{1}>0$, such that for any $0 \leq v \leq v_{1}$, we have

$$
\left|I+v \partial_{j} a I I\right|>M_{1}>0 .
$$

When $\operatorname{Re} \tilde{s}=0$ with $\tilde{s}^{2} \neq \omega^{2}\left(u_{+}-\sigma\right)^{2}$ and $\tilde{s} \neq 0$, we know that if $\tilde{s}^{2}$ does not equal to the right hand side of (51) with the minus sign, then the inequality (52) holds for any $0 \leq v \leq v_{1}$ with sufficiently small $v_{1}>0$. If $\tilde{s}^{2}$ satisfies (51) with the minus sign, then the term $I$ vanishes. However, the imaginary part of the term II given in (49) is nonzero, which implies that for any fixed $v>0$, there is a constant $M_{1}>0$ such that the inequality (52) holds as well.

Therefore, we can find $v_{1}>0$ and $K_{1}>0$ depending on $U_{+}$and $\sigma$ given in (10) and the constant $\beta$ given in (18) such that $\mathcal{L}_{1}$ does not vanish for $0<v \leq v_{1}$ and $0 \leq K \leq K_{1}$.

2) $\tilde{s}^{2}=\omega^{2}\left(u_{+}-\sigma\right)^{2}$.

In this case, we get

$$
\begin{aligned}
& \mathcal{L}_{1}= \pm 8 i c_{+} j^{3}[\tau]\left(\sqrt[+]{\tilde{s}^{2}+\omega^{2} d_{-}^{2}}+\frac{\tau_{+}}{\tau_{-}} \omega c_{-}\right)+O(1) \tilde{\gamma} \\
& \text { for } \pm \omega>0 .
\end{aligned}
$$

which is nonzero for sufficiently small $v>0$ and $K>0$.

3) $\tilde{s}=0$.

In this case, we have

$$
\mathcal{L}_{1}=\frac{4 d_{+} d_{-} j}{c_{+} c_{-} \tau_{+} \tau_{-}}\left(j \tau_{+}[\tau]-\tilde{\gamma}\right)
$$

which is also nonzero for sufficiently small $v>0$ and $K>0$.

Therefore, combining the above arguments, we draw the conclusion of the Theorem 4.1.

\section{REFERENCES}

[1] S.-Y. Zhang, "Existence of Travelling Waves in NonIsothermal Phase Dynamics," Journal of Hyperbolic Differential Equations, Vol. 3, No. 4, 2007, pp. 391-400. doi:10.1142/S0219891607001197

[2] H. Fan and M. Slemrod, "Dynamic Flows with Liquid/ Vapor Phase Transitions," In: H. Fan and M. Slemrod, Eds., Handbook of Mathematical Fluid Dynamics, Vol. I, North-Holland, Amsterdam, 2002, pp. 373-420.

[3] P. D. Lax, "Hyperbolic Systems of Conservation Laws. II," Communications on Pure and Applied Mathematics, Vol. 10, 1957, pp. 537-467. doi:10.1002/cpa.3160100406

[4] M. Slemrod, "Admissibility Criteria for Propagating Phase Boundaries in a Van Der Waals Fluid," Archive for Rational Mechanics and Analysis, Vol. 81, No. 4, 1983, pp. 301-315.

[5] M. Shearer, "Nonuniqueness of Admissible Solutions of Riemann Initial Value Problem for a System of Conser- 
vation Laws of Mixed Type," Archive for Rational Mechanics and Analysis, Vol. 93, No. 1, 1986, pp. 45-59. doi:10.1007/BF00250844

[6] P. G. LeFloch, "Propagating Phase Boundaries: Formulation of the Problem and Existence via the Glimm Method," Archive for Rational Mechanics and Analysis, Vol. 123, No. 2, 1993, pp. 153-197. doi:10.1007/BF00695275

[7] S. Benzoni-Gavage, "Stability of Multi-Dimensional Phase Transitions in a Van Der Waals Fluid," Nonlinear Analysis, Vol. 31, No. 1-2, 1998, pp. 243-263. doi:10.1016/S0362-546X(96)00309-4

[8] S. Benzoni-Gavage, "Stability of Subsonic Planar Phase Boundaries in a Van Der Waals Fluid," Archive for Rational Mechanics and Analysis, Vol. 150, No. 1, 1999, pp. 23-55. doi:10.1007/s002050050179

[9] Y.-G. Wang and Z. Xin, "Stability and Existence of Multidimensional Subsonic Phase Transitions," Acta Mathematicae Applicatae Sinica. English Series, Vol. 19, No. 4, 2003, pp. 529-558.

[10] S.-Y. Zhang, "Discontinuous Solutions to the Euler Equations in a Van Der Waals Fluid," Applied Mathematics
Letters, Vol. 20, No. 2, 2007, pp. 170-176. doi:10.1016/j.aml.2006.03.010

[11] M. Slemrod, "Dynamic Phase Transitions in a Van Der Waals Fluid," Journal of Differential Equations, Vol. 52, No. 1, 1984, pp. 1-23. doi:10.1016/0022-0396(84)90130-X

[12] M. Grinfeld, "Nonisothermal Dynamic Phase Transitions," Quarterly of Applied Mathematics, Vol. 47, No. 1, 1989, pp. 71-84.

[13] H. Hattori, “The Riemann Problem for a Van Der Waals fluid with Entropy Rate Admissibility Criterion Nonisothermal Case," Journal of Differential Equations, Vol. 65, No. 2, 1986, pp. 158-174. doi:10.1016/0022-0396(86)90031-8

[14] H. O. Kreiss, "Initial Boundary Value Problems for Hyperbolic Systems," Communications on Pure and Applied Mathematics, Vol. 23, 1970, pp. 227-298. doi:10.1002/cpa.3160230304

[15] A. Majda, "The Stability of Multi-Dimensional Shock Fronts," Memoirs of the American Mathematical Society, Vol. 41, No. 275, 1983, pp. 1-95. 